\title{
Research on the New Direction of Artificial Intelligence Development Writer
}

\author{
Zhezhou Kang ${ }^{1, \text { a }}$ \\ ${ }^{1}$ Beijing Jiaotong University \\ a 542156367@qq.com
}

Keywords: Artificial Intelligence; Development direction; Research

\begin{abstract}
Artificial intelligence is now well known to more and more people. Especially, AlphaGo won world go champion Leeshishi. And more and more families are using vacuum cleaners. In the development of artificial intelligence, there are different kinds of voices in society. Actually, there are also a lot of shortcomings. And that inevitably leads us to think about how to develop artificial intelligence, to study the new direction of development, to find a solution.
\end{abstract}

\section{Artificial Intelligence}

The concept of artificial intelligence. Artificial intelligence can be divided into "artificial" and "intelligent" parts. Artificial intelligence has two important foundations: one is the degree to which the structural man is intelligent; Second, the construction of intelligent technology. So, artificial intelligence is based on the basis of studying the law of human intelligence activity, to construct a intelligent system similar to human intelligence activity, through the development of computer technology, make the person's intelligence is qualified for the job or thinking activity have a computer, simulate the human way of thinking, behavior, and other functions of science and technology.

The Development of Artificial Intelligence. In the 1950s, the advent of computers, humans began to really step into the use of technology to simulate human intelligence, to simulate human thought. At the time, there were also some preliminary intelligences that used electronic networks, such as w. GREY WALTER's TURTLES and JOHNS HOPKINS BEAST.

In the early 1960s, researchers focused on developing expert systems that led to advances in artificial intelligence.

In the 1980s, Japan began the "fifth generation computer development program", which led to the study of artificial intelligence and entered a period of intensive development. Then, neural network, driven by the United States, international conference, promote the rapid development of the research of neural network, greatly improve the efficiency of data processing, provides conditions for subsequent development of artificial intelligence.

International Internet technology in the $1990 \mathrm{~s}$ into the mature stage of development and application, also promotes the present artificial intelligence on the shift from single line thinking to line $3 \mathrm{~d}$, this also invisible to expand the application range of artificial intelligence.

The development of science and technology is changing, from the concept of artificial intelligence to AlphaGo defeat human these big events, the development of artificial intelligence has been beyond our expectations. From computer learning has become a compulsory course, it's not hard to see, the importance of the development of science and technology, and artificial intelligence has always been the forefront of computer science disciplines, so the development of artificial intelligence is beyond imagination. 


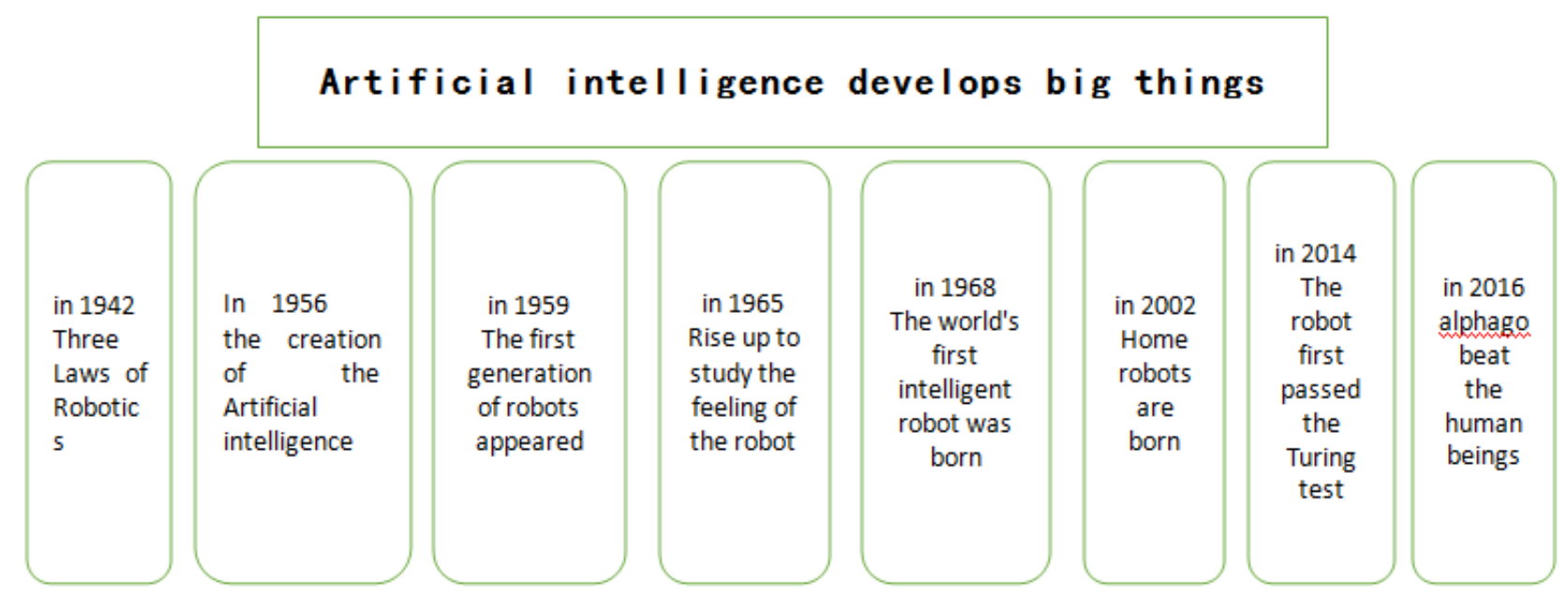

The Value and Significance of Artificial Intelligence Development. Artificial intelligence is the technology that collects and compilts data from computer technology to match human intelligence. At the core is a large amount of data base, input data, and output instructions or information to achieve some kind of functionality. It is important to see how the data is transmitted.

Nowadays, the development of the society, whether the development of the economy, the progress of the society, has been carried forward with the complete information of The Times. To start a business, know the market capacity information, product information, customer information... The more information people have, the more likely they are to do so. Similar laws of information used in HangHangYeYe, artificial intelligence is nothing but essentially is the carrier of information, the artificial intelligence industry, and other fields has a positive role in promoting.

In addition, the emergence of artificial intelligence, instead of some human labor, liberated the hands of the working people, realized certain conjugate energy. For example, face card player, voice control system, sweeping robot... All kinds of artificial intelligence, which bring different forms to our lives, provide more convenient and convenient options.

The emergence of artificial intelligence and the development and progress of the computer industry. The computer industry is constantly being upgraded because of the stringent requirements of cutting-edge technology.

The emergence of artificial intelligence partly opens a new chapter in human activity. Such as the development of VR technology, which not only increases the people's enjoyment of the quality of life, but also broadens the way of life science and technology.

The Development and Bottleneck of Artificial Intelligence.. After so many years of development, artificial intelligence is also on the higher step, was in the lab research object, has now entered the people's ordinary life. Artificial intelligence realizes the reality of people's service through its own function. Even so, there are plenty of problems with the development of artificial intelligence.

First, it is limited to the level of research on the brain's own intelligence. The current research on brain activity is incomplete. Simple to imitate the structure and function of the human brain, though is likely to be the correct research direction, but the human brain structure, our team of working mechanism of the human brain is not yet known deep comprehensive.

Secondly, there are many problems in the technical level, whether it is data integrity collection, or precision expression. This limits the presentation and presentation of artificial intelligence research.

Moreover, it is limited to the level of research of existing ai researchers. The creation of artificial intelligence is not simply a sum, nor is it a simple chemistry experiment. It is a leap forward and has many uncertainties. Nowadays artificial development is limited by the knowledge level, scientific 
research ability and even imagination ability of the existing talent team.

In short, although we have made strides in artificial intelligence, this step is only a small step in the history of artificial intelligence development. Things can't be smooth sailing, there are difficulties, there is a problem, is a necessary process, now that they had found the problem, how and when we do a good job in the next step is the key.

\section{Thinking About the New Direction of Artificial Intelligence.}

According to the above analysis, we will begin to think about the new direction of artificial intelligence development. By definition, we took the artificial intelligence is divided into "human" and "smart" two parts, so in order to solve the existing development bottleneck shall also be considered from the two aspects.

"Artificial" Developing New Direction of Thinking. The article also mentions the development of artificial intelligence first in the understanding of the structure and mechanism of human brain, and the influence of human research level. For artificial intelligence, it's all external factors. So how to deal with avoidance. Bold ideas, whether can make the existing artificial intelligence in a separate "I", the role of an independent "self-consciousness", by artificial intelligence "ego" to complete the set goals, to complete the task, self assessment, improve the process of self-development.

Based on the objective of the appeal, we must introduce the target element in the study of artificial intelligence and set the target parameters for artificial intelligence. The instructions of the action and the effects of the action all have the effect of instructing the "self" to do what it needs to accomplish. Through such research, the constant revision of the target parameters makes artificial intelligence itself more relevant to the human being, not simply a performer of an algorithm.

"Intelligence" Develops New Direction of Thinking. Smart development focuses on functional implementation. During the analysis of the problem, we also found the structure and mechanism of the human brain in the development of artificial intelligence. The structure is easy to imitate and is weak in the exploration of the mechanism. Cannot be realized and the structure of the simply imitate the function of the human body itself is through the mechanism of human brain in command, to achieve a certain functional, structure only material carrier, can realize the function of the few and far between.

In addition, there are still many occur in the brain mechanism of imitate reality barriers: first of all, humanity itself to explore the mechanism of the human brain is not enough comprehensive, copied foundation is weak, so even if imitation, also cannot too. Second, in a timely manner in the near future, we thoroughly understanding of the mechanism of the human brain, carefully researched, and then we have no technology can completely copy and construction; Moreover, on the premise of both possess in time, we also have to think, in the replication of static imitation is only a temporary, only dynamic changes in the last. Finally, the problem of information sources, artificial intelligence is from passive collection, is the state of the input and the experience of not its own, is unfavorable to the development of for a long time.

In short, in the development of intelligence, it is still possible to search for ideas from within, to find breakthrough, to really push the further development of artificial intelligence.

\section{Artificial Intelligence Development Trend Forecast}

Service Human. Artificial intelligence is created out of the need to meet the human needs. Artificial intelligence is achieved primarily through its functional research into the purpose of human services. The earliest artificial intelligence and human beings were used for industrial production, and now the sweeping robots are also serving the daily life of human beings. Shift from the need to meet the needs of the production workforce to the demands of a smarter life. Human beings are becoming more and more diverse in the function of artificial intelligence. It also makes the development of artificial intelligence more and more relevant, and more and more relevant to the human self. Such trends will also become more prominent in the development of artificial 
intelligence.

Functional Replacement Human. The development of many industries in life is destructive to human beings. The chemical industry, for example, is highly polluting. For the environment, the human blow is devastating. The development of artificial intelligence, to some extent, can solve this problem, the pollution of intelligent manufacturing system, intelligent processing systems, intelligent water operating system, the human in the whole process only needs to play the role of supervision, well behind the eyes.

Artificial Intelligence and Human Equality. There are more and more science fiction movies about artificial intelligence, and more and more people are thinking about developing artificial intelligence. Some fear of being replaced by fear of being replaced, or by imagining the future of artificial intelligence. Have such psychological is normal, but should not be replaced, if you want to have the bright future of artificial intelligence, can with our own learning and potential, so as to realize the artificial intelligence in dealing with the equality of human beings.

We Will Explore the Margin of Artificial Intelligence. Artificial intelligence can serve human beings on the one hand, and on the other hand, it can also be explored by its own exploration. Artificial factors are external, and they will certainly limit the development of artificial intelligence. Artificial intelligence is the development direction, and if it can realize its own exploration, it is not too far away from the true artificial intelligence.

\section{Conclusion.}

According to the text content, the new development direction of the future of artificial intelligence is from "human" and "smart" two angles, one is the realization of the functional sex of the artificial intelligence is more and more close to real life; The second is to gradually eliminate the influence of external labor, and make it "self-aware". It is believed that with our continuous exploration, we will really build the artificial intelligence friendly society, bring many conveniences to our work and life.

\section{References:}

[1] Chen qingxia. Development process and prospect of artificial intelligence research program [J]. Scientific information, 2008 (33)

[2] yi-xin zhong. The breakthrough and the innovation of the scientific method of artificial intelligence $[\mathrm{J}]$. Journal of pattern recognition and artificial intelligence, 2012, 25 (3): 456. 456

[3] shang fuhua, li jun, wang mei, et al. Artificial intelligence and its application [M]. Beijing: petroleum industry press, 2005

[4] sun ye, wu fei.research status and development trends of artificial intelligence. [J]. Human resources management. Value engineering. 2013 (28) : 5-7

[5] wedrian. The dilemma of artificial intelligence development [D]. Nanning: guangxi normal university. 2009 